\title{
ESSENTIAL NORMALITY AND THE DECOMPOSABILITY OF HOMOGENEOUS SUBMODULES
}

\author{
MATTHEW KENNEDY
}

\begin{abstract}
We establish the essential normality of a large new class of homogeneous submodules of the finite rank $d$-shift Hilbert module. The main idea is a notion of essential decomposability that determines when a submodule can be decomposed into the algebraic sum of essentially normal submodules. We prove that every essentially decomposable submodule is essentially normal, and introduce methods for establishing that a submodule is essentially decomposable. It turns out that many submodules have this property. We prove that many of the submodules considered by other authors are essentially decomposable, and in addition establish the essential decomposability of a large new class of homogeneous submodules. Our results support Arveson's conjecture that every homogeneous submodule of the finite rank $d$-shift Hilbert module is essentially normal.
\end{abstract}

\section{INTRODUCTION}

In this paper we establish new results in higher-dimensional operator theory that support Arveson's conjecture of a corresponence between algebraic varieties and $\mathrm{C}^{*}$-algebras of essentially normal operators. Specifically, we prove the essential normality of a large new class of homogeneous submodules of the finite rank $d$-shift Hilbert module introduced by Arveson in Arv98. Our work provides a new perspective on Arveson's conjecture that every homogeneous submodule is essentially normal.

For fixed $d \geq 1$, let $\mathbb{C}[z]=\mathbb{C}\left[z_{1}, \ldots, z_{d}\right]$ denote the algebra of complex polynomials in $d$ variables. With the introduction of an appropriate inner product, $\mathbb{C}[z]$ can be completed to a space of analytic functions on the complex unit ball called the Drury-Arveson space, which we denote by $H_{d}^{2}$. The coordinate multiplication operators $M_{z_{1}}, \ldots, M_{z_{d}}$, defined on $\mathbb{C}[z]$ by

$$
\left(M_{z_{i}} p\right)\left(z_{1}, \ldots, z_{d}\right)=z_{i} p\left(z_{1}, \ldots, z_{d}\right), \quad p \in \mathbb{C}[z], 1 \leq i \leq d,
$$

extend to bounded linear operators on $H_{d}^{2}$, and $\left(M_{z_{1}}, \ldots, M_{z_{d}}\right)$ forms a contractive $d$-tuple of operators called the $d$-shift. The space $H_{d}^{2}$ can be naturally viewed as a module over $\mathbb{C}[z]$, with the module action given by

$$
p f=p\left(M_{z_{1}}, \ldots, M_{z_{d}}\right) f, \quad p \in \mathbb{C}[z], f \in H_{d}^{2} .
$$

Endowed with this module action, $H_{d}^{2}$ is called the $d$-shift Hilbert module.

The $d$-shift and the $d$-shift Hilbert module $H_{d}^{2}$ are of fundamental importance in multivariable operator theory, and they have received a great deal of attention in recent years (see for example [Arv98, Arv00, Arv02, Arv05, Arv07, DRS11, [DRS12], Dou06], GW08, Esc11, Sha11]).

Received by the editors May 25, 2012 and, in revised form, December 17, 2012.

2010 Mathematics Subject Classification. Primary 47A13, 47A20, 47A99, 14Q99, 12 Y05. 
Let $M$ be a submodule of $H_{d}^{2}$, so that $M$ is an invariant subspace for each coordinate operator $M_{z_{1}}, \ldots, M_{z_{d}}$. If we identify the quotient space $H_{d}^{2} / M$ with the orthogonal complement $M^{\perp}$, then we obtain a $d$-tuple of quotient operators $\left(T_{1}, \ldots, T_{d}\right)$ by compressing the $d$-shift $\left(M_{z_{1}}, \ldots, M_{z_{d}}\right)$ to $H_{d}^{2} / M$. Since the operators $M_{z_{1}}, \ldots, M_{z_{d}}$ commute, the operators $T_{1}, \ldots, T_{d}$ also commute, and in fact, every commuting contractive $d$-tuple of operators can be realized as a quotient of the $d$-shift in precisely this way, provided that one is willing to increase the multiplicity and consider vector-valued functions (see for example Arv98).

The quotient module $H_{d}^{2} / M$ is said to be p-essentially normal if the selfcommutators

$$
T_{i}^{*} T_{j}-T_{j} T_{i}^{*}, \quad 1 \leq i, j \leq d
$$

belong to the Schatten $p$-class $\mathcal{L}^{p}$ for $1 \leq p \leq \infty$ (where $\mathcal{L}^{\infty}$ denotes the ideal of compact operators $\mathcal{K})$. We also obtain a $d$-tuple of operators $\left(S_{1}, \ldots, S_{d}\right)$ by restricting the elements in the $d$-shift $\left(M_{z_{1}}, \ldots, M_{z_{d}}\right)$ to $M$, and the module $M$ is similarly said to be $p$-essentially normal if the self-commutators

$$
S_{i}^{*} S_{j}-S_{j} S_{i}^{*}, \quad 1 \leq i, j \leq d
$$

belong to $\mathcal{L}^{p}$ for $1 \leq p \leq \infty$. In fact, it turns out that these notions of essential normality are equivalent for $p>d$, since the submodule $M$ is $p$-essentially normal if and only if the quotient module $H_{d}^{2} / M$ is $p$-essentially normal (see for example Arv05).

The purpose of this paper is to consider the essential normality of submodules of the $d$-shift Hilbert module $H_{d}^{2}$, and more generally, the essential normality of submodules of the finite rank $d$-shift Hilbert module $H_{d}^{2} \otimes \mathbb{C}^{r}$, obtained by tensoring $H_{d}^{2}$ with $\mathbb{C}^{r}$, for a positive integer $r \geq 1$. Note that elements in $H_{d}^{2} \otimes \mathbb{C}^{r}$ can be viewed as analytic vector-valued functions on the complex unit ball.

Arveson observed in Arv02 that $H_{d}^{2} \otimes \mathbb{C}^{r}$ is itself $p$-essentially normal for every $p>d$, and motivated by applications to multivariable Fredholm Theory, he asked whether every homogeneous submodule of the finite rank $d$-shift Hilbert module, i.e. every submodule generated by homogeneous polynomials, is $p$-essentially normal for every $p>d$.

In Arv05, Arveson conjectured that this question should have an affirmative answer, and established the truth of his conjecture for submodules of $H_{d}^{2} \otimes \mathbb{C}^{r}$ generated by monomials, i.e. polynomials of the form $z_{1}^{\alpha_{1}} \cdots z_{d}^{\alpha_{d}} \otimes \xi$ for $\alpha=\left(\alpha_{1}, \ldots, \alpha_{d}\right)$ in $\mathbb{N}_{0}^{d}$ and $\xi$ in $\mathbb{C}$. More recently, in GW08, Guo and Wang proved that every submodule of $H_{d}^{2} \otimes \mathbb{C}^{r}$ generated by a single homogeneous polynomial is $p$-essentially normal for every $p>d$. Additionally, they proved that for $d \leq 3$, every homogeneous submodule of $H_{d}^{2} \otimes \mathbb{C}^{r}$ is $p$-essentially normal for every $p>d$. However, none of these results apply to homogeneous submodules of $H_{d}^{2} \otimes \mathbb{C}^{r}$ when $d \geq 4$ and the submodule is generated by two or more non-monomials.

In this paper, we establish Arveson's conjecture for a large new class of homogeneous submodules of the finite-rank $d$-shift Hilbert module. This class includes homogeneous submodules of $H_{d}^{2} \otimes \mathbb{C}^{r}$, with $d$ arbitrarily large, that are generated by an arbitrary number of non-monomials. For example, we obtain the following result.

Theorem 1.1. Let $F_{1}, \ldots, F_{n}$ be sets of homogeneous polynomials in $\mathbb{C}\left[z_{1}, \ldots, z_{d}\right]$. Suppose that there are disjoint subsets $Z_{1}, \ldots, Z_{n}$ of $\left\{z_{1}, \ldots, z_{d}\right\}$, each of size at 
most 2, such that

$$
F_{i} \subseteq \mathbb{C}\left[Z_{i}\right], \quad 1 \leq i \leq n
$$

Let $X_{1}, \ldots, X_{n}$ be arbitrary sets of vectors in $\mathbb{C}^{r}$. Then the $H_{d}^{2} \otimes \mathbb{C}^{r}$ submodule generated by the set of vector-valued polynomials

$$
\left\{p \otimes \xi \mid p \in F_{i}, \xi \in X_{i}, 1 \leq i \leq n\right\}
$$

is $p$-essentially normal for every $p>d$.

We obtain Theorem 1.1 as a special case of the following more broadly applicable result.

Theorem 1.2. Let $F_{1}, \ldots, F_{n}$ be sets of polynomials in $\mathbb{C}\left[z_{1}, \ldots, z_{d}\right]$ that each generate p-essentially normal submodules of $H_{d}^{2}$. Suppose that there are disjoint subsets $Z_{1}, \ldots, Z_{n}$ of $\left\{z_{1}, \ldots, z_{d}\right\}$, such that

$$
F_{i} \subseteq \mathbb{C}\left[Z_{i}\right], \quad 1 \leq i \leq n
$$

Let $X_{1}, \ldots, X_{n}$ be arbitrary sets of vectors in $\mathbb{C}^{r}$. Then the $H_{d}^{2} \otimes \mathbb{C}^{r}$ submodule generated by the set of vector-valued polynomials

$$
\left\{p \otimes \xi \mid p \in F_{i}, \xi \in X_{i}, 1 \leq i \leq n\right\}
$$

is $p$-essentially normal for every $p>d$.

More generally, we obtain Theorem 1.2 as an application of a new method for establishing the essential normality of a submodule of $H_{d}^{2} \otimes \mathbb{C}^{r}$. First, we observe that if $M_{1}, \ldots, M_{n}$ are $p$-essentially normal submodules of $H_{d}^{2} \otimes \mathbb{C}^{r}$ with the property that the algebraic sum $M_{1}+\ldots+M_{n}$ is closed, then the $H_{d}^{2} \otimes \mathbb{C}^{r}$ submodule generated by $M_{1}, \ldots, M_{n}$ is also $p$-essentially normal. Reversing this argument tells us that if we want to prove the $p$-essential normality of an $H_{d}^{2} \otimes \mathbb{C}^{r}$ submodule $M$, then we should try to obtain a decomposition of $M$ as $M=M_{1}+\ldots+M_{n}$, where $M_{1}, \ldots, M_{n}$ are $p$-essentially normal submodules of $H_{d}^{2} \otimes \mathbb{C}^{r}$.

Note that by Guo and Wang's result on the essential normality of submodules generated by a single homogeneous polynomial, every homogeneous submodule of $H_{d}^{2} \otimes \mathbb{C}^{r}$ can be written as a closed sum of essentially normal submodules. Therefore, the main difficulty is understanding when, if ever, the algebraic sum $M_{1}+\ldots+M_{n}$ is closed. While this problem seems quite difficult in general, we prove below that this sum is closed in many interesting cases.

Our results also imply the essential normality of submodules that have been considered by other authors. For example, our results imply the essential decomposability of submodules of $H_{d}^{2} \otimes \mathbb{C}^{r}$ generated by monomials, and so we obtain a new proof of Arveson's main result in Arv05.

In addition to this introduction, this paper has five other sections. In Section 2 , we provide a brief review of the basic background material. In Section 3, we introduce the notion of essential decomposability, and relate it to Shalit's stable division property from Sha11. In Section 4, we introduce a notion of perpendicularity for a family of submodules that implies essential decomposability. In Section 5 , we establish the main results on essential normality. 


\section{Preliminaries}

2.1. The $d$-shift Hilbert module. For fixed $d \geq 1$, let $\mathbb{C}[z]=\mathbb{C}\left[z_{1}, \ldots, z_{d}\right]$ denote the algebra of complex polynomials in $d$ variables. For monomials in $\mathbb{C}[z]$, it is convenient to use the multi-index notation

$$
z^{\alpha}=z_{1}^{\alpha_{1}} \cdots z_{d}^{\alpha_{d}}, \quad \alpha=\left(\alpha_{1}, \ldots, \alpha_{d}\right) \in \mathbb{N}_{0}^{d} .
$$

The Drury-Arveson space $H_{d}^{2}$ is the completion of $\mathbb{C}[z]$ with respect to the inner product $\langle\cdot, \cdot\rangle$, defined on monomials by

$$
\left\langle z^{\alpha}, z^{\beta}\right\rangle=\delta_{\alpha \beta} \frac{\alpha !}{|\alpha| !}, \quad \alpha, \beta \in \mathbb{N}_{0}^{d},
$$

where we have written $\alpha !=\alpha_{1} ! \cdots \alpha_{d}$ ! and $|\alpha|=\alpha_{1}+\ldots+\alpha_{d}$ for $\alpha=\left(\alpha_{1}, \ldots, \alpha_{d}\right)$ in $\mathbb{N}_{0}^{d}$.

Let $M_{z_{1}}, \ldots, M_{z_{d}}$ denote the coordinate multiplication operators on $\mathbb{C}[z]$ corresponding to the variables $z_{1}, \ldots, z_{d}$ respectively,

$$
M_{z_{i}} p=z_{i} p, \quad p \in \mathbb{C}[z], 1 \leq i \leq d .
$$

Then these operators extend to bounded linear operators on $H_{d}^{2}$, and the $d$-tuple $\left(M_{z_{1}}, \ldots, M_{z_{d}}\right)$ is called the $d$-shift.

The elements in $H_{d}^{2}$ can be identified with analytic functions on the complex unit ball, and $H_{d}^{2}$ can be viewed as a Hilbert module over the algebra of polynomials $\mathbb{C}[z]$, with the module action defined by

$$
p f=p\left(M_{z_{1}}, \ldots, M_{z_{d}}\right) f, \quad p \in \mathbb{C}[z], f \in H_{d}^{2} .
$$

Endowed with this module action, $H_{d}^{2}$ is called the $d$-shift Hilbert module. The importance of this construction in multivariable operator theory was recognized by Arveson in his comprehensive treatment Arv98.

Let $N$ denote the number operator, the unbounded self-adjoint operator defined on monomials in $\mathbb{C}[z]$ by

$$
N z^{\alpha}=|\alpha| z^{\alpha}, \quad \alpha \in \mathbb{N}_{0}^{d},
$$

and extended to polynomials in $\mathbb{C}[z]$ by linearity. Then the operator $(N+1)^{-1}$ extends to a bounded operator on $H_{d}^{2}$. Let $\partial_{1}, \ldots, \partial_{d}$ denote the operators that act on $\mathbb{C}[z]$ by partial differentiation with respect to the variables $z_{1}, \ldots, z_{d}$ respectively. Then restricted to $\mathbb{C}[z]$, we can write

$$
M_{z_{i}}^{*}=(N+1)^{-1} \partial_{i}, \quad 1 \leq i \leq d .
$$

The fact that the adjoint operators take this form is one reason for the importance of the $d$-shift (see Arv98 for details).

More generally, for a polynomial $p$ in $\mathbb{C}[z]$, let $M_{p}$ denote the operator on $H_{d}^{2}$ corresponding to multiplication by $p$,

$$
M_{p} f=p f, \quad f \in H_{d}^{2} .
$$

2.2. The finite rank $d$-shift Hilbert module. We will need to consider a higher multiplicity version of $H_{d}^{2}$. For fixed $r \geq 1$, the $d$-shift Hilbert module of rank $r$, $H_{d}^{2} \otimes \mathbb{C}^{r}$, is the Hilbert space tensor product of $H_{d}^{2}$ with the $r$-dimensional Hilbert space $\mathbb{C}^{r}$. Note that we could also realize $H_{d}^{2} \otimes \mathbb{C}^{r}$ as the completion of the algebraic tensor product $\mathbb{C}[z] \otimes \mathbb{C}^{r}$. We will follow Arv07, and write $r \mathbb{C}[z]$ and $r H_{d}^{2}$ for $\mathbb{C}[z] \otimes \mathbb{C}^{r}$ and $H_{d}^{2} \otimes \mathbb{C}^{r}$ respectively. 
Since the meaning will always be clear from the context, it will be convenient to also let $M_{z_{1}}, \ldots, M_{z_{d}}$ denote the coordinate multiplication operators on $r H_{d}^{2}$,

$$
M_{z_{i}}(f \otimes \xi)=M_{z_{i}} f \otimes \xi, \quad f \in H_{d}^{2}, \xi \in \mathbb{C}^{r}, 1 \leq i \leq d .
$$

Note that cordinate multiplication operators on $r H_{d}^{2}$ can also be realized as the tensor product of the coordinate multiplication operators on $H_{d}^{2}$ with the identity operator on $\mathbb{C}^{r}$. The $d$-tuple $\left(M_{z_{1}}, \ldots, M_{z_{d}}\right)$ is called the $d$-shift of rank $r$.

The elements in $r H_{d}^{2}$ can be viewed as vector-valued analytic functions on the complex unit ball, and $r H_{d}^{2}$ can also be viewed as a Hilbert module over the algebra of polynomials $\mathbb{C}[z]$. In this case, the module action is defined on the elementary tensors in $r H_{d}^{2}$ by

$$
p(f \otimes \xi)=p\left(M_{z_{1}}, \ldots, M_{z_{d}}\right)(f \otimes \xi), \quad p \in \mathbb{C}[z], f \in H_{d}^{2}, \xi \in \mathbb{C}^{r},
$$

and extended to all of $r H_{d}^{2}$ by linearity.

2.3. Essential normality. Let $N$ be a submodule of $r H_{d}^{2}$. Then $N$ is invariant for the coordinate multiplication operators $M_{z_{1}}, \ldots, M_{z_{d}}$ on $r H_{d}^{2}$, so we can consider the corresponding restrictions $S_{1}, \ldots, S_{d}$ to $N$. The submodule $N$ is said to be p-essentially normal if the self-commutators

$$
S_{i}^{*} S_{j}-S_{j} S_{i}^{*}, \quad 1 \leq i, j \leq d,
$$

belong to the Schatten $p$-class $\mathcal{L}^{p}$ for $1 \leq p \leq \infty$. If $p=\infty$, then we will say that $N$ is essentially normal.

If we identify the quotient space $r H_{d}^{2} / N$ with the orthogonal complement $N^{\perp}$, then we can also consider the compressions $T_{1}, \ldots, T_{d}$ of $M_{z_{1}}, \ldots, M_{z_{d}}$ respectively to $r H_{d}^{2} / N$. The quotient module $r H_{d}^{2} / N$ is similarly said to be $p$-essentially normal if the self-commutators

$$
T_{i}^{*} T_{j}-T_{j} T_{i}^{*}, \quad 1 \leq i, j \leq d,
$$

belong to the Schatten class $\mathcal{L}^{p}$ for $1 \leq p \leq \infty$.

For a submodule $N$, let $P_{N}$ denote the projection onto $N$. We will require the following result of Arveson, which is Theorem 4.3 of Arv05.

Theorem 2.1 (Arveson). Let $N$ be a submodule of $r H_{d}^{2}$. Then for every $p>d$, the following are equivalent:

(1) $N$ is p-essentially normal,

(2) $r H_{d}^{2} / N$ is p-essentially normal,

(3) for $1 \leq i \leq d$, the commutators $M_{z_{i}} P_{N}-P_{N} M_{z_{i}}$ belong to the Schatten p-class $\mathcal{L}^{2 p}$.

In our work, we will mostly use condition (3) of Theorem 2.1.

\section{EsSEnTIAL DECOMPOSABILITY}

3.1. Essential decomposability. In this section, we will consider a notion of decomposability for a submodule that implies essential normality. Let $N_{1}, \ldots, N_{n}$ be submodules of $r H_{d}^{2}$. Then we will write $N_{1}+\ldots+N_{n}$ for the (not necessarily closed) algebraic sum

$$
N_{1}+\ldots+N_{n}=\left\{x_{1}+\ldots+x_{n} \mid x_{i} \in N_{i} \text { for } 1 \leq i \leq n\right\} .
$$


Definition 3.1. Let $N$ be a submodule of $r H_{d}^{2}$. Then $N$ is said to be $p$-essentially decomposable if there are $p$-essentially normal $r H_{d}^{2}$ submodules $N_{1}, \ldots, N_{n}$ such that

$$
N=N_{1}+\ldots+N_{n}
$$

If $p=\infty$, then $N$ is said to be essentially decomposable.

Remark 3.2. Note that if $N$ is a $p$-essentially normal submodule of $r H_{d}^{2}$, then it is trivially $p$-essentially decomposable.

Theorem 3.3. Every $p$-essentially decomposable submodule of $r H_{d}^{2}$ is p-essentially normal for $p>d$.

Proof. Let $N$ be a $p$-essentially decomposable submodule of $r H_{d}^{2}$ with decomposition $N=N_{1}+\ldots+N_{n}$, where $N_{1}, \ldots, N_{n}$ are $p$-essentially normal $r H_{d}^{2}$ submodules. Let $M=N_{1} \oplus \ldots \oplus N_{n}$. Then $M$ is a submodule of $n r H_{d}^{2}=\left(r H_{d}^{2}\right)^{n}$, and the $p$ essential normality of $N_{1}, \ldots, N_{n}$ implies the $p$-essential normality of $M$. Define $L:\left(r H_{d}^{2}\right)^{n} \rightarrow r H_{d}^{2}$ by

$$
L\left(x_{1}, \ldots, x_{n}\right)=x_{1}+\ldots+x_{n}, \quad\left(x_{1}, \ldots, x_{n}\right) \in\left(r H_{d}^{2}\right)^{n} .
$$

Then $L\left(M_{z_{i}}^{(n)}\right)^{*}=M_{z_{i}}^{*} L$ for each $i$, where $M_{z_{i}}^{(n)}$ denotes the coordinate multiplication operator on $\left(r H_{d}^{2}\right)^{n}$. In particular, the restriction of $L$ to $M$ is a $2 p$ morphism from $\left(r H_{d}^{2}\right)^{n}$ to $r H_{d}^{2}$, in the sense of Definition 4.3 of Arv07. Furthermore, $L(M)=N$ is closed. Since $M$ is $p$-essentially normal, it follows from Theorem 4.4 of Arv07 that $N$ is also $p$-essentially normal.

Remark 3.4. A direct proof of Theorem 3.3 that avoids the notion of a $p$-morphism can be given by emulating the first part of the proof of Theorem 4.4 of Arv07.

We will also require the following lemma, which can be proved by a simple modification of the proof of Corollary 3 of [FW71].

Lemma 3.5. Let $N_{1}, \ldots, N_{n}$ be submodules of $r H_{d}^{2}$. Then the algebraic sum $N_{1}+$ $\ldots+N_{n}$ is closed if and only if the range of the operator $P_{N_{1}}+\ldots+P_{N_{n}}$ is closed.

Remark 3.6. A classical result of Friedrichs [Fri37] implies that the algebraic sum of two subspaces $N_{1}$ and $N_{2}$ is closed if and only if the (Friedrichs) angle between them is positive. Recently in [BGM10, Badea, Grivaux and Müller established an analogue of Friedrichs' result for an arbitrary number of subspaces $N_{1}, \ldots, N_{n}$, by considering a generalized notion of angle. Although we do not require their results in the present paper, we believe that similar ideas may eventually prove useful in resolving Arveson's conjecture.

3.2. Stable division. The stable division property was introduced by Shalit in Sha11, in connection with Arveson's conjecture. However, the notion of stable division is also of independent interest, since it concerns the numerical stability of multivariable polynomial division.

Definition 3.7. An $r H_{d}^{2}$ submodule $N$ is said to have the stable division property if there is a family of homogeneous polynomials $\left\{p_{1}, \ldots, p_{n}\right\}$ generating $N$ and a constant $C \geq 0$ such that, for any polynomial $p$ in $N$, there are polynomials $q_{1}, \ldots, q_{n}$ in $\mathbb{C}[z]$ satisfying $p=q_{1} p_{1}+\ldots+q_{n} p_{n}$ and

$$
\left\|q_{1} p_{1}\right\|+\ldots+\left\|q_{n} p_{n}\right\| \leq C\|p\| .
$$

The family $\left\{p_{1}, \ldots, p_{n}\right\}$ is said to be a stable generating set for $N$. 
The next result was discovered at the suggestion of Shalit. It establishes a connection between the ideas in this paper and his work on the stable division property in Sha11.

Theorem 3.8. Let $p_{1}, \ldots, p_{n}$ be homogeneous polynomials in $r \mathbb{C}[z]$, and let $N_{1}$, $\ldots, N_{n}$ denote the corresponding $r H_{d}^{2}$ submodules they generate. Then $\left\{p_{1}, \ldots, p_{n}\right\}$ is a stable generating set if and only if the algebraic sum $N_{1}+\ldots+N_{n}$ is closed.

Proof. Let $N=\overline{N_{1}+\ldots+N_{n}}$. Suppose first that $N_{1}+\ldots+N_{n}$ is closed. Then the operator $T: N_{1} \oplus \ldots \oplus N_{n} \rightarrow N$, defined by

$$
T\left(x_{1}, \ldots, x_{n}\right)=x_{1}+\ldots+x_{n}, \quad\left(x_{1}, \ldots, x_{n}\right) \in N_{1} \oplus \ldots \oplus N_{n},
$$

has closed range $N$. Hence there is a constant $C \geq 0$ such that for any $f$ in $N$, there are $f_{i}$ in $N_{i}$ satisfying $f=f_{1}+\ldots+f_{n}$ and

$$
\left\|f_{1}\right\|+\ldots+\left\|f_{n}\right\| \leq C\|f\| .
$$

If $f=p$ is a homogeneous polynomial, then we can replace each $f_{i}$ with the homogeneous polynomial $q_{i}^{\prime}$ obtained by projecting $f_{i}$ onto the homogeneous component of $r H_{d}^{2}$ containing $p$. Since $N_{i}$ is generated by the homogeneous polynomial $p_{i}$, it is left invariant by this projection. Hence $q_{i}^{\prime}$ still belongs to $N_{i}$, and we can write $q_{i}^{\prime}=q_{i} p_{i}$ for some polynomial $q_{i}$ in $\mathbb{C}[z]$. Since an arbitrary polynomial can be written as an orthogonal sum of homogeneous polynomials of different degrees, it follows that $\left\{p_{1}, \ldots, p_{n}\right\}$ is a stable generating set for $N$.

Conversely, suppose $\left\{p_{1}, \ldots, p_{n}\right\}$ is a stable generating set for $N$. Fix $f$ in $N$, and let $\left(s_{k}\right)_{k=1}^{\infty}$ be a sequence of polynomials in $N$ converging to $f$. By the stable division property, there is a constant $C \geq 0$ such that, for each $k \geq 1$, there are polynomials $q_{k, i}$ in $\mathbb{C}[z]$ satisfying $s_{k}=q_{k, 1} p_{1}+\ldots+q_{k, n} p_{n}$ and

$$
\left\|q_{k, 1} p_{1}\right\|+\ldots+\left\|q_{k, n} p_{n}\right\| \leq C\left\|s_{k}\right\| .
$$

For each $i$, the sequence $\left(q_{k, i} p_{i}\right)_{k=1}^{\infty}$ is clearly bounded, and by passing to a subsequence we can assume that it is weakly convergent to some $f_{i}$ in $N_{i}$. Then for all $g$ in $r H_{d}^{2}$,

$$
\left\langle f-\left(f_{1}+\ldots+f_{n}\right), g\right\rangle=\lim _{k \rightarrow \infty}\left\langle s_{k}-\left(q_{k, 1} p_{1}+\ldots+q_{k, n} p_{n}\right), g\right\rangle=0,
$$

and it follows that $f=f_{1}+\ldots+f_{k}$. Hence $f$ belongs to $N_{1}+\ldots+N_{n}$. Since $f$ was arbitrary, we conclude that $N=N_{1}+\ldots+N_{n}$.

Shalit proved in Theorem 2.3 of Sha11 that many families of homogeneous submodules of $H_{2}^{2}$ have the stable division property. However, consideration of Shalit's proof reveals that it actually implies the following stronger result. (For background material on Groebner bases, see, for example, [CLS92.)

Theorem 3.9 (Shalit). Let $p_{1}, \ldots, p_{n}$ be homogeneous polynomials in $\mathbb{C}[z]$ such that $\left\{p_{1}, \ldots, p_{n}\right\}$ is a Groebner basis. Suppose there is a subset $Z$ of $\left\{z_{1}, \ldots, z_{d}\right\}$, of size at most 2 , such that $p_{1}, \ldots, p_{n} \in \mathbb{C}[Z]$. Then the family $\left\{p_{1}, \ldots, p_{n}\right\}$ is a stable generating set.

Applying Theorem 3.8 to Theorem 3.9, we obtain the following result.

Proposition 3.10. Let $N_{1}, \ldots, N_{n}$ be submodules of $H_{d}^{2}$ generated by homogeneous polynomials $p_{1}, \ldots, p_{n}$ respectively in $\mathbb{C}[z]$. Suppose that $\left\{p_{1}, \ldots, p_{n}\right\}$ is a Groebner basis, and suppose that there is a subset $Z$ of $\left\{z_{1}, \ldots, z_{d}\right\}$, of size at most 2 , such that $p_{1}, \ldots, p_{n} \in \mathbb{C}[Z]$. Then the algebraic sum $N_{1}+\ldots+N_{n}$ is closed. 
Applying Theorem 3.8 to an example from Sha11 provides an example of two submodules of $H_{d}^{2}$ with non-closed algebraic sum, and demonstrates that there is no straightforward generalization of Proposition 3.10 to polynomials in three or more variables.

Example 3.11. Let $N_{1}$ and $N_{2}$ denote the $H_{3}^{2}$ submodules generated by the polynomials $p_{1}$ and $p_{2}$ respectively, where

$$
\begin{aligned}
& p_{1}\left(z_{1}, z_{2}, z_{3}\right)=z_{1}^{2}+z_{2} z_{3} \\
& p_{2}\left(z_{1}, z_{2}, z_{3}\right)=z_{2}^{2},
\end{aligned}
$$

and let $N=\overline{N_{1}+N_{2}}$. In Example 2.6 of Sha11 it was shown that the family $\left\{p_{1}, p_{2}\right\}$ generates $N$, but is not a stable generating set. Hence by Theorem 3.8 , the algebraic sum $N_{1}+N_{2}$ is not closed. Since $\left\{p_{1}, p_{2}\right\}$ is a Groebner basis with respect to the lexicographical monomial ordering, this also shows that Theorem 3.8 does not generalize to polynomials in three or more variables.

However, $N$ is essentially decomposable. Indeed, let $K_{1}, K_{2}, K_{3}, K_{4}$ denote the submodules of $H_{3}^{2}$ generated by the polynomials $q_{1}, q_{2}, q_{3}, q_{4}$ respectively, where

$$
\begin{aligned}
& q_{1}\left(z_{1}, z_{2}, z_{3}\right)=z_{1}^{4} \\
& q_{2}\left(z_{1}, z_{2}, z_{3}\right)=z_{1}^{2} z_{2} \\
& q_{3}\left(z_{1}, z_{2}, z_{3}\right)=z_{1}^{2}+z_{2} z_{3} \\
& q_{4}\left(z_{1}, z_{2}, z_{3}\right)=z_{2}^{2} .
\end{aligned}
$$

Then the family $\left\{q_{1}, q_{2}, q_{3}, q_{4}\right\}$ is a stable generating set for $N$. Hence by Theorem 3.8, $N=K_{1}+K_{2}+K_{3}+K_{4}$.

We also briefly mention Eschmeier's recent paper [Esc11, which introduces a related property of a family of polynomials, in connection with Arveson's essential normality conjecture. In fact, as pointed out in [Sha11, Eschmeier's property is implied by the stable division property.

\section{Perpendicular submodules}

4.1. Perpendicularity. In this section we consider a property of a family of submodules of $r H_{d}^{2}$ that implies the algebraic sum of the submodules is closed.

Definition 4.1. Let $N_{1}, \ldots, N_{n}$ be submodules of $r H_{d}^{2}$. The family $\left\{N_{1}, \ldots, N_{n}\right\}$ is perpendicular if

$$
N_{i} \cap\left(N_{i} \cap N_{j}\right)^{\perp} \perp N_{j} \cap\left(N_{i} \cap N_{j}\right)^{\perp}, \quad 1 \leq i<j \leq n .
$$

Proposition 4.2. Let $N_{1}, \ldots, N_{n}$ be submodules of $r H_{d}^{2}$. Then the family $\left\{N_{1}, \ldots\right.$, $\left.N_{n}\right\}$ is perpendicular if and only if the projections $P_{N_{1}}, \ldots, P_{N_{n}}$ commute.

Proof. We recall the simple fact that if $P$ and $Q$ are projections with range $\operatorname{ran}(P)$ and $\operatorname{ran}(Q)$ respectively, then $P$ and $Q$ commute if and only if

$$
\operatorname{ran}(P) \cap(\operatorname{ran}(P) \cap \operatorname{ran}(Q))^{\perp} \perp \operatorname{ran}(Q) \cap(\operatorname{ran}(P) \cap \operatorname{ran}(Q))^{\perp} .
$$

Therefore, the result follows immediately from Definition 4.1 ,

Ken Davidson pointed out that the following lemma is a well-known result from the theory of CSL (commutative subspace lattice) algebras. 
Lemma 4.3. Let $\left\{N_{1}, \ldots, N_{n}\right\}$ be a perpendicular family of submodules of $r H_{d}^{2}$, and let $K_{1}, \ldots, K_{m}$ be subspaces contained in the subspace lattice generated by $N_{1}, \ldots, N_{n}$. Then $\left\{K_{1}, \ldots, K_{m}\right\}$ is also a perpendicular family of submodules of $r H_{d}^{2}$.

Proof. The projections $P_{K_{1}}, \ldots, P_{K_{n}}$ are contained in the von Neumann algebra generated by the projections $P_{N_{1}}, \ldots, P_{N_{n}}$, and by Proposition 4.2, this von Neumann algebra is commutative. In particular, the projections $P_{K_{1}}, \ldots, P_{K_{n}}$ commute, and another application of Proposition 4.2 implies that the family $\left\{K_{1}, \ldots\right.$, $\left.K_{m}\right\}$ is perpendicular.

4.2. Criteria for perpendicularity. In this section, we will consider criteria for a family of submodules to be perpendicular.

Lemma 4.4. Let $N_{1}, \ldots, N_{n}$ be submodules of $H_{d}^{2}$, and for $1 \leq i \leq n$, let $p_{i 1}, \ldots$, $p_{i m_{i}}$ be polynomials that generate $N_{i}$. If the operators

$$
M_{p_{i 1}} M_{p_{i 1}}^{*}+\ldots+M_{p_{i m_{i}}} M_{p_{i m_{i}}}^{*}, \quad 1 \leq i \leq n
$$

commute, then the family $\left\{N_{1}, \ldots, N_{n}\right\}$ is perpendicular.

Proof. For $1 \leq i, j \leq n, P_{N_{i}}$ and $P_{N_{j}}$ are the range projections of the operators $M_{p_{i 1}} M_{p_{i 1}}^{*}+\ldots+M_{p_{i m_{i}}} M_{p_{i m_{i}}}^{*}$ and $M_{p_{j 1}} M_{p_{j 1}}^{*}+\ldots+M_{p_{j m_{j}}} M_{p_{j m_{j}}}^{*}$ respectively. In particular, the projections $P_{N_{i}}$ and $P_{N_{j}}$ are contained in the von Neumann algebra generated by $M_{p_{i 1}} M_{p_{i 1}}^{*}+\ldots+M_{p_{i m_{i}}} M_{p_{i m_{i}}}^{*}$ and $M_{p_{j 1}} M_{p_{j 1}}^{*}+\ldots+M_{p_{j m_{j}}} M_{p_{j m_{j}}}^{*}$. Since these latter operators are self-adjoint, and since they commute, this von Neumann algebra is commutative, meaning in particular that the projections $P_{N_{i}}$ and $P_{N_{j}}$ commute. Since $i$ and $j$ were arbitrary, Proposition 4.2 implies that the family $\left\{N_{1}, \ldots, N_{n}\right\}$ is perpendicular.

To apply Lemma 4.4, we will require an identity of Guo and Wang from GW08. Before presenting the identity, it will be convenient to introduce some special notation for operators that are related to the number operator $N$ defined in Section 2.1. For a function $f: \mathbb{Z} \rightarrow \mathbb{Z}$, let $[f(N)]$ denote the (potentially unbounded) self-adjoint operator defined on monomials in $\mathbb{C}[z]$ by

$$
[f(N)] z^{\alpha}=f(|\alpha|) z^{\alpha}, \quad \alpha \in \mathbb{N}_{0}^{d},
$$

and extended by linearity to polynomials in $\mathbb{C}[z]$. Then, for example, restricted to $\mathbb{C}[z]$, we can write the adjoints of the coordinate multiplication operators $M_{z_{1}}^{*}, \ldots$, $M_{z_{d}}^{*}$ on $H_{d}^{2}$ as

$$
M_{z_{i}}^{*}=\left[\frac{1}{N+1}\right] \partial_{i}, \quad 1 \leq i \leq d,
$$

where $\partial_{1}, \ldots, \partial_{d}$ denote the operators that act on $\mathbb{C}[z]$ by partial differentiation in the variable $z_{1}, \ldots, z_{d}$ respectively. If the operator $[f(N)]$ happens to extend to a bounded operator on $H_{d}^{2}$, then we will also write $[f(N)]$ for this extension. Recall that for a polynomial $p$ in $\mathbb{C}[z]$, we write $M_{p}$ to denote the operator on $H_{d}^{2}$ corresponding to multiplication by $p$. If $p$ is homogeneous of degree $n$, then it is easy to check that, restricted to $\mathbb{C}[z]$, we can write

$$
[f(N)] M_{p}=M_{p}[f(N+n)] .
$$


These facts, combined with the general Leibniz rule

$$
\partial^{\alpha}(p q)=\sum_{\substack{\beta \in \mathbb{N}_{0}^{d} \\
\beta \leq \alpha}}\left(\begin{array}{l}
\alpha \\
\beta
\end{array}\right)\left(\partial^{\alpha-\beta} p\right)\left(\partial^{\beta} q\right), \quad \alpha \in \mathbb{N}_{0}^{d}, p, q \in \mathbb{C}[z],
$$

where $\partial^{\alpha}=\partial_{\alpha_{1}} \cdots \partial_{\alpha_{d}}$, lead to the following identity of Guo and Wang from GW08.

Proposition 4.5 (Guo-Wang identity). Let $p$ and $q$ be homogeneous polynomials in $\mathbb{C}[z]$ of degree $m$ and $n$ respectively. Then

$$
M_{p}^{*} M_{q}=\sum_{\alpha \in \mathbb{N}_{0}^{d}} \frac{1}{\alpha !}\left[\frac{N !(N+m-n) !}{(N+m) !(N-n+|\alpha|) !}\right] M_{\partial^{\alpha} q} M_{\partial^{\alpha} p}^{*} .
$$

We will apply Proposition 4.5 to determine when the hypotheses of Lemma 4.4 hold.

Lemma 4.6. Let $p$ and $q$ be homogeneous polynomials in $\mathbb{C}[z]$ of degree $m$ and $n$ respectively. Then

$$
\begin{aligned}
M_{p} & M_{p}^{*} M_{q} M_{q}^{*}-M_{q} M_{q}^{*} M_{p} M_{p}^{*} \\
& =\sum_{\alpha \in \mathbb{N}_{0}^{d} \backslash\{0\}} \frac{1}{\alpha !}\left[\frac{(N-m) !(N-n) !}{N !(N-m-n+|\alpha|) !}\right]\left(M_{p} M_{\partial^{\alpha} q} M_{q}^{*} M_{\partial^{\alpha} p}^{*}-M_{\partial^{\alpha} p} M_{q} M_{\partial^{\alpha} q}^{*} M_{p}^{*}\right) .
\end{aligned}
$$

Proof. The Guo-Wang identity from Proposition 4.5 gives

$$
\begin{aligned}
M_{p} M_{p}^{*} M_{q} M_{q}^{*} & =\sum_{\alpha \in \mathbb{N}_{0}^{d}} M_{p} \frac{1}{\alpha !}\left[\frac{N !(N+m-n) !}{(N+m) !(N-n+|\alpha|) !}\right] M_{\partial^{\alpha} q} M_{\partial^{\alpha} p}^{*} M_{q}^{*} \\
& =\sum_{\alpha \in \mathbb{N}_{0}^{d}} \frac{1}{\alpha !}\left[\frac{(N-m) !(N-n) !}{N !(N-m-n+|\alpha|) !}\right] M_{p} M_{\partial^{\alpha} q} M_{q}^{*} M_{\partial^{\alpha} p}^{*},
\end{aligned}
$$

and by symmetry this implies

$$
M_{q} M_{q}^{*} M_{p} M_{p}^{*}=\sum_{\alpha \in \mathbb{N}_{0}^{d}} \frac{1}{\alpha !}\left[\frac{(N-m) !(N-n) !}{N !(N-m-n+|\alpha|) !}\right] M_{\partial^{\alpha} p} M_{q} M_{\partial^{\alpha} q}^{*} M_{p}^{*} .
$$

The result now follows by taking the difference of these identities.

Lemma 4.7. Let $p$ and $q$ be homogeneous polynomials in $\mathbb{C}[z]$ of degree $m$ and $n$ respectively. Then $M_{p} M_{p}^{*}$ and $M_{q} M_{q}^{*}$ commute if and only if the operator

$$
\sum_{\alpha \in \mathbb{N}_{0}^{d} \backslash\{0\}} \frac{1}{\alpha !}\left[\frac{(N-m) !(N-n) !}{N !(N-m-n-|\alpha|) !}\right] M_{p} M_{\partial^{\alpha} q} M_{q}^{*} M_{\partial^{\alpha} p}^{*}
$$

is self-adjoint.

Proof. This follows immediately from Lemma 4.6, using the observation that for $\alpha$ in $\mathbb{N}_{0}^{d}$,

$$
\begin{aligned}
M_{p} M_{\partial^{\alpha} q} M_{q}^{*} M_{\partial^{\alpha} p}^{*} & -M_{\partial^{\alpha} p} M_{q} M_{\partial^{\alpha} q}^{*} M_{p}^{*} \\
& =M_{p} M_{\partial^{\alpha} q} M_{q}^{*} M_{\partial^{\alpha} p}^{*}-\left(M_{p} M_{\partial^{\alpha} q} M_{q}^{*} M_{\partial^{\alpha} p}^{*}\right)^{*} .
\end{aligned}
$$


Lemma 4.8. Let $p$ and $q$ be homogeneous polynomials in $\mathbb{C}[z]$ of degree $m$ and $n$ respectively. Then $M_{p} M_{p}^{*}$ and $M_{q} M_{q}^{*}$ commute if each operator

$$
M_{p} M_{\partial^{\alpha} q} M_{q}^{*} M_{\partial^{\alpha} p}^{*}, \quad \alpha \in \mathbb{N}_{0}^{d} \backslash\{0\}
$$

is self-adjoint.

Proof. This follows immediately from Lemma 4.7

Lemma 4.9. Let $p$ and $q$ be linear polynomials in $\mathbb{C}[z]$. Then the operators $M_{p} M_{P}^{*}$ and $M_{q} M_{q}^{*}$ commute if either $p=q$ or $p \perp q$.

Proof. Write the polynomials $p$ and $q$ as

$$
\begin{aligned}
& p\left(z_{1}, \ldots, z_{d}\right)=a_{1} z_{1}+\ldots+a_{d} z_{d} \\
& q\left(z_{1}, \ldots, z_{d}\right)=b_{1} z_{1}+\ldots+b_{d} z_{d} .
\end{aligned}
$$

Then

$$
\begin{aligned}
\sum_{\alpha \in \mathbb{N}_{0}^{d} \backslash\{0\}} \frac{1}{\alpha !}\left[\frac{(N-m) !(N-n) !}{N !(N-m-n+|\alpha|) !}\right] & M_{p} M_{\partial^{\alpha} q} M_{q}^{*} M_{\partial^{\alpha} p}^{*} \\
& =\sum_{i=1}^{d}\left[\frac{(N-1) !(N-1) !}{N !(N+1) !}\right] a_{i} \overline{b_{i}} M_{p} M_{q}^{*} \\
& =\langle p, q\rangle\left[\frac{(N-1) !(N-1) !}{N !(N+1) !}\right] M_{p} M_{q}^{*} .
\end{aligned}
$$

Hence the operator (4.2) is self-adjoint if either $p=q$ or $p \perp q$, and the result follows by Lemma 4.7 .

Lemma 4.10. Let $z^{\lambda}$ and $z^{\mu}$ be monomials in $\mathbb{C}[z]$ for $\lambda$ and $\mu$ in $\mathbb{N}_{0}^{d}$. Then the operators $M_{z^{\lambda}} M_{z^{\lambda}}^{*}$ and $M_{z^{\mu}} M_{z^{\mu}}^{*}$ commute.

Proof. For $\alpha$ in $\mathbb{N}_{0}^{d}$,

$$
z^{\lambda}\left(\partial^{\alpha} z^{\mu}\right)=c_{\mu} z^{\lambda_{1}+\mu_{1}-\alpha_{1}} \cdots z^{\lambda_{d}+\mu_{d}-\alpha_{d}},
$$

where

$$
c_{\mu}= \begin{cases}\prod_{i=1}^{d} \mu_{i}\left(\mu_{i}-1\right) \cdots\left(\mu_{i}-\alpha_{i}+1\right) & \text { if } \alpha_{i} \leq \mu_{i} \text { for } 1 \leq i \leq d, \\ 0 & \text { otherwise. }\end{cases}
$$

Similarly,

$$
\left(\partial^{\alpha} z^{\lambda}\right) z^{\mu}=c_{\lambda} z^{\lambda_{1}+\mu_{1}-\alpha_{1}} \cdots z^{\lambda_{d}+\mu_{d}-\alpha_{d}}
$$

where

$$
c_{\lambda}= \begin{cases}\prod_{i=1}^{d} \lambda_{i}\left(\lambda_{i}-1\right) \cdots\left(\lambda_{i}-\alpha_{i}+1\right) & \text { if } \alpha_{i} \leq \lambda_{i} \text { for } 1 \leq i \leq d, \\ 0 & \text { otherwise. }\end{cases}
$$

Let $\nu=\left(\lambda_{1}+\mu_{1}-\alpha_{1}, \ldots, \lambda_{d}+\mu_{d}-\alpha_{d}\right)$. Then

$$
M_{z^{\lambda}} M_{\partial^{\alpha} z^{\mu}} M_{z^{\mu}}^{*} M_{\partial^{\alpha} z^{\lambda}}^{*}= \begin{cases}c_{\lambda} c_{\mu} M_{z^{\nu}} M_{z^{\nu}}^{*} & \text { if } \nu \in \mathbb{N}_{0}^{d}, \\ 0 & \text { otherwise. }\end{cases}
$$

In particular, this operator is self-adjoint. Therefore, by Lemma 4.8, the operators $M_{z^{\lambda}} M_{z^{\lambda}}^{*}$ and $M_{z^{\mu}} M_{z^{\mu}}^{*}$ commute. 
Lemma 4.11. Let $p$ and $q$ be homogeneous in $\mathbb{C}[z]$ in distinct variables. Then the operators $M_{p} M_{p}^{*}$ and $M_{q} M_{q}^{*}$ commute.

Proof. Since $p$ and $q$ are polynomials in disjoint variables, for every $\alpha \in \mathbb{N}_{0}^{d} \backslash\{0\}$, at least one of $\partial^{\alpha} p$ and $\partial^{\alpha} q$ must be zero, and hence at least one of $M_{\partial^{\alpha} p}$ and $M_{\partial^{\alpha}} q$ must be zero. In particular, this implies that $M_{p} M_{\partial^{\alpha} q} M_{q}^{*} M_{\partial^{\alpha} p}^{*}=0$, and it follows from Lemma 4.8 that $M_{p} M_{p}^{*}$ and $M_{q} M_{q}^{*}$ commute.

4.3. Perpendicular submodules. In this section, we will establish the perpendicularity of many families of submodules of $H_{d}^{2}$ using the criteria from Section 4.2 .

Proposition 4.12. Let $N_{1}, \ldots, N_{n}$ be submodules of $H_{d}^{2}$ that are generated by mutually orthogonal sets of linear polynomials $F_{1}, \ldots, F_{n}$ respectively. Then the family $\left\{N_{1}, \ldots, N_{n}\right\}$ is perpendicular.

Proof. This follows immediately from Lemma 4.4 and Proposition 4.2

Proposition 4.13. Let $N_{1}, \ldots, N_{n}$ be submodules of $H_{d}^{2}$ each generated by monomials. Then the family $\left\{N_{1}, \ldots, N_{n}\right\}$ is perpendicular.

Proof. This follows immediately from Lemma 4.4 and Lemma 4.10.

Proposition 4.14. Let $N_{1}, \ldots, N_{n}$ be submodules of $H_{d}^{2}$ generated by sets of homogeneous polynomials $F_{1}, \ldots, F_{n}$ respectively. Suppose that there are disjoint subsets $Z_{1}, \ldots, Z_{n}$ of $\left\{z_{1}, \ldots, z_{d}\right\}$ such that

$$
F_{i} \subseteq \mathbb{C}\left[Z_{i}\right], \quad 1 \leq i \leq n .
$$

Then the family $\left\{N_{1}, \ldots, N_{n}\right\}$ is perpendicular.

Proof. This follows immediately from Lemma 4.4 and Lemma 4.11.

We can strengthen Proposition 4.14 using results of Carlini and Reznick. The following result is Lemma 3.1 in Rez93].

Proposition 4.15 (Rez93). Let $p_{1}, \ldots, p_{n}$ be homogeneous polynomials in $\mathbb{C}[z]$. If the sets

$$
\left\{\partial_{z^{\alpha}} p_{i}|| \alpha \mid=\operatorname{deg}\left(p_{i}\right)-1, \alpha \in \mathbb{N}_{0}^{d}\right\}, \quad 1 \leq i \leq n,
$$

are mutually orthogonal, then there is a unitary change of variables such that the polynomials $p_{1}, \ldots, p_{n}$ are polynomials in disjoint variables.

The following result is Proposition 1 in [Car06].

Proposition 4.16 (Car06). Let $p_{1}, \ldots, p_{n}$ be homogeneous polynomials in $\mathbb{C}[z]$. If the sets

$$
\left\{\nabla p_{i}(z) \mid z \in \mathbb{C}^{d}\right\}, \quad 1 \leq i \leq n,
$$

are mutually orthogonal, where $\nabla p$ denotes the gradient of $p$, then there is a unitary change of variables such that the polynomials $p_{1}, \ldots, p_{n}$ are polynomials in disjoint variables.

We immediately obtain the following two results.

Proposition 4.17. Let $N_{1}, \ldots, N_{n}$ be submodules of $H_{d}^{2}$ generated by sets of homogeneous polynomials $F_{1}, \ldots, F_{n}$ respectively. If the sets

$$
\left\{\partial^{\alpha}(p)|| \alpha \mid=\operatorname{deg}(p)-1, \alpha \in \mathbb{N}_{0}^{d}, p \in F_{i}\right\}, \quad 1 \leq i \leq n,
$$

are mutually orthogonal, then the family $\left\{N_{1}, \ldots, N_{n}\right\}$ is perpendicular. 
Proposition 4.18. Let $N_{1}, \ldots, N_{n}$ be submodules of $H_{d}^{2}$ generated by sets of homogeneous polynomials $F_{1}, \ldots, F_{n}$ respectively. If the sets

$$
\left\{(\nabla p)(\lambda) \mid \lambda \in \mathbb{C}^{d}, p \in F_{i}\right\}, \quad 1 \leq i \leq n,
$$

are mutually orthogonal, then the family $\left\{N_{1}, \ldots, N_{n}\right\}$ is perpendicular.

4.4. Perpendicularity and tensor products. The results obtained in Section 4.2 and Section 4.3 only apply to submodules of $H_{d}^{2}$. Because we also need to consider higher-rank submodules of $r H_{d}^{2}$, in this section we consider tensor products of perpendicular submodules.

Theorem 4.19. Let $\left\{N_{1}, \ldots, N_{n}\right\}$ be a perpendicular family of submodules of $H_{d}^{2}$, and let $V_{1}, \ldots V_{n}$ be arbitrary subspaces of $\mathbb{C}^{r}$. Let $M_{1}, \ldots, M_{n}$ denote the $r H_{d}^{2}$ submodules

$$
M_{i}=N_{i} \otimes V_{i}, \quad 1 \leq i \leq n .
$$

Then the algebraic sum $M_{1}+\ldots+M_{n}$ is closed.

Proof. Let $E_{1}, \ldots, E_{n}$ denote the projections onto $V_{1}, \ldots, V_{n}$ respectively. Then it's clear that

$$
P_{M_{i}}=P_{N_{i}} \otimes E_{i}, \quad 1 \leq i \leq n .
$$

We will prove that the operator

$$
P_{M_{1}}+\ldots+P_{M_{n}}
$$

has closed range. By Lemma 3.5, this will imply the desired result.

We proceed by induction on $n$, the size of the family $\left\{N_{1}, \ldots, N_{n}\right\}$. For $n=1$, the result is trivially true. Therefore, suppose that $n \geq 2$ with the induction hypothesis that the result is true for a perpendicular family of submodules of $H_{d}^{2}$ if the size of the family is at most $n-1$.

Let $Q_{0}, \ldots, Q_{n}$ denote the projections onto $r H_{d}^{2}$ defined by

$$
\begin{aligned}
Q_{0} & =P_{N_{1}} \cdots P_{N_{n}} \otimes I \\
Q_{1} & =P_{N_{1}}^{\perp} \otimes I \\
Q_{2} & =P_{N_{1}} P_{N_{2}}^{\perp} \otimes I \\
& \vdots \\
Q_{n} & =P_{N_{1}} \cdots P_{N_{n-1}} P_{N_{n}}^{\perp} \otimes I .
\end{aligned}
$$

Since the family $\left\{N_{1}, \ldots, N_{n}\right\}$ is perpendicular, Proposition 4.2 implies that the projections $P_{N_{1}}, \ldots, P_{N_{n}}$ commute, and hence that the projections $Q_{0}, \ldots, Q_{n}$ also commute. It's also clear that

$$
Q_{i} P_{M_{j}}=P_{M_{j}} Q_{i}, \quad 1 \leq i, j \leq n .
$$

Furthermore, by construction the projections $Q_{0}, \ldots, Q_{n}$ are orthogonal,

$$
Q_{i} Q_{j}=0, \quad 0 \leq i<j \leq n,
$$

and we can decompose the identity operator on $r H_{d}^{2}$ as

$$
I=Q_{0}+Q_{1}+Q_{2}+\ldots+Q_{n} .
$$


Therefore, by (4.3), (4.4) and (4.5), we can write

$$
\begin{aligned}
P_{M_{1}}+\ldots+P_{M_{n}} & =\left(\sum_{i=0}^{n} Q_{i}\right)\left(\sum_{j=1}^{n} P_{M_{j}}\right)\left(\sum_{i=0}^{m} Q_{i}\right) \\
& =\sum_{i=0}^{n} \sum_{j=0}^{n} Q_{i} P_{M_{j}} .
\end{aligned}
$$

Now, for $1 \leq j \leq n$,

$$
\begin{aligned}
Q_{0} P_{M_{j}} & =\left(P_{N_{1}} \cdots P_{N_{n}} \otimes I\right)\left(P_{N_{j}} \otimes E_{j}\right) \\
& =P_{N_{1}} \cdots P_{N_{n}} \otimes E_{j} \\
& =Q_{0}\left(I \otimes E_{j}\right)
\end{aligned}
$$

and this gives

$$
Q_{0}\left(\sum_{j=1}^{n} P_{M_{j}}\right)=Q_{0} \sum_{j=1}^{n}\left(I \otimes E_{j}\right) .
$$

By a similar calculation, for $1 \leq i \leq n$,

$$
\begin{aligned}
Q_{i} P_{M_{i}} & =\left(P_{N_{1}} \cdots P_{N_{i-1}} P_{N_{i}}^{\perp} \otimes I\right)\left(P_{N_{i}} \otimes E_{i}\right) \\
& =P_{N_{1}} \cdots P_{N_{i-1}} P_{N_{i}}^{\perp} P_{N_{i}} \otimes E_{i} \\
& =0
\end{aligned}
$$

and this gives

$$
Q_{i} \sum_{j=1}^{n} P_{M_{j}}=Q_{i} \sum_{\substack{j=1 \\ j \neq i}}^{n} P_{M_{j}}
$$

Let $X_{0}$ denote the operator on $r H_{d}^{2}$ defined by

$$
X_{0}=I \otimes \sum_{j=1}^{n} E_{j}
$$

and let $X_{1}, \ldots, X_{n}$ denote the operators on $H_{d}^{2} \otimes \mathbb{C}^{r}$ defined by

$$
X_{i}=\sum_{\substack{j=1 \\ j \neq i}}^{n} P_{M_{j}}, \quad 1 \leq i \leq n .
$$

Then by the induction hypothesis and Lemma 3.5, the operators $X_{0}, \ldots, X_{n}$ each have closed range, and by (4.6), (4.7) and (4.8), we can write

$$
P_{M_{1}}+\ldots+P_{M_{n}}=Q_{0} X_{0}+\ldots+Q_{n} X_{n} .
$$

Since the operators $X_{0}, \ldots, X_{n}$ commute with the projections $Q_{0}, \ldots, Q_{n}$, it follows that the operators $Q_{0} X_{0}, \ldots, Q_{n} X_{n}$ each have closed range. Therefore, since the projections $Q_{0}, \ldots, Q_{n}$ are orthogonal, this means that we have written $P_{M_{1}}+\ldots+$ $P_{M_{n}}$ as the direct sum of $n+1$ operators that each have closed range. It follows that the range of this operator is also closed. 


\section{EsSential NORMALity}

\subsection{Essential normality and perpendicularity.}

Lemma 5.1. Let $N$ be a $p$-essentially normal submodule of $H_{d}^{2}$ for $p>d$, and let $V$ be an arbitrary subspace of $\mathbb{C}^{r}$. Then the $r H_{d}^{2}$ submodule $N \otimes V$ is also p-essentially normal.

Proof. Let $E$ denote the projection onto $V$, and let $M=N \otimes V$. Then it's clear that

$$
P_{M}=P_{N} \otimes E \text {. }
$$

By Theorem 2.1, the $p$-essential normality of $N$ implies that the projection $P_{N} 2 p$ essentially commutes with the coordinate multiplication operators $M_{z_{1}}, \ldots, M_{z_{d}}$ on $H_{d}^{2}$, i.e.

$$
M_{z_{i}} P_{N}-P_{N} M_{z_{i}} \in \mathcal{L}^{2 p}, \quad 1 \leq i \leq d,
$$

where $\mathcal{L}^{2 p}$ denotes the set of Schatten $2 p$-class operators on $H_{d}^{2}$. Recall from Section 2.2 that we can write the coordinate multiplication operators on $r H_{d}^{2}$ as $M_{z_{1}} \otimes$ $I, \ldots, M_{z_{d}} \otimes I$. Hence for $1 \leq i \leq d$,

$$
\begin{aligned}
\left(M_{z_{i}} \otimes I\right) P_{M}-P_{M}\left(M_{z_{i}} \otimes I\right) & =\left(M_{z_{i}} \otimes I\right)\left(P_{N} \otimes E\right)-\left(P_{N} \otimes E\right)\left(M_{z_{i}} \otimes I\right) \\
& =\left(M_{z_{i}} P_{N}-P_{N} M_{z_{i}}\right) \otimes E \in \mathcal{L}^{2 p},
\end{aligned}
$$

since $E$ is a finite rank projection. Therefore, by Theorem 2.1, $M$ is $2 p$-essentially normal.

Theorem 5.2. Let $\left\{N_{1}, \ldots, N_{n}\right\}$ be a perpendicular family of p-essentially decomposable submodules of $H_{d}^{2}$ for $p>d$, and let $V_{1}, \ldots, V_{n}$ be arbitrary subspaces of $\mathbb{C}^{r}$. Let $M_{1}, \ldots, M_{n}$ denote the $r H_{d}^{2}$ submodules

$$
M_{i}=N_{i} \otimes V_{i}, \quad 1 \leq i \leq n .
$$

Then the $\mathrm{rH}_{d}^{2}$ submodule $\overline{M_{1}+\ldots+M_{n}}$ is also p-essentially normal.

Proof. By Lemma 5.1 the submodules $M_{1}, \ldots, M_{n}$ are $p$-essentially normal. Let $M$ denote the $r H_{d}^{2}$ submodule $M=\overline{M_{1}+\ldots+M_{n}}$. By Theorem 4.19, we can write $M=M_{1}+\ldots+M_{n}$. It follows that $M$ is $p$-essentially decomposable, and hence by Theorem 3.3 that $M$ is $p$-essentially normal.

5.2. Essential normality. In this section, we establish our main results on the essential normality of homogeneous submodules of $r H_{d}^{2}$. We will require Guo and Wang's result, Theorem 2.2 from [GW08, about the essential normality of singly generated homogeneous submodules.

Theorem 5.3 (Guo-Wang). Every submodule of $r H_{d}^{2}$ generated by a single homogeneous polynomial is $p$-essentially normal for every $p>d$.

The next result is well known. It was proved, for example, by Shalit in Sha11], using his results on stable division. The methods introduced here provide a new proof.

Theorem 5.4. Every submodule of $H_{d}^{2}$ generated by linear polynomials is $p$ essentially normal for every $p>d$. 
Proof. Let $N$ be a submodule of $H_{d}^{2}$ generated by linear polynomials $p_{1}, \ldots, p_{n}$ in $\mathbb{C}[z]$. By applying the Gram-Schmidt process if necessary, we can assume that the set $\left\{p_{1}, \ldots, p_{n}\right\}$ is orthogonal in $H_{d}^{2}$. Let $N_{1}, \ldots, N_{n}$ denote the $H_{d}^{2}$ submodules generated by $p_{1}, \ldots, p_{n}$ respectively. Then Theorem 5.3 implies that these submodules are each $p$-essentially normal for every $p>d$, and Proposition 4.12 implies that the family $\left\{N_{1}, \ldots, N_{n}\right\}$ is perpendicular. Note that $N=\overline{N_{1}+\ldots+N_{n}}$. Hence by Theorem 5.2 $N$ is also $p$-essentially normal for every $p>d$.

The next result is new. It establishes the essential normality of submodules of $r H_{d}^{2}$ that are generated by certain linear polynomials. We note Arveson's result from [Arv07] that the problem of the essential normality of homogeneous submodules of $r H_{d}^{2}$ is equivalent to the problem of the essential normality of submodules of $r H_{d}^{2}$ generated by arbitrary linear polynomials.

Theorem 5.5. Let $F_{1}, \ldots, F_{n}$ be mutually orthogonal sets of linear polynomials, and let $X_{1}, \ldots, X_{n}$ be arbitrary sets of vectors in $\mathbb{C}^{r}$. Then the $r H_{d}^{2}$ submodule generated by the set of vector-valued polynomials

$$
\left\{p \otimes \xi \mid p \in F_{i}, \xi \in X_{i}, 1 \leq i \leq n\right\}
$$

is $p$-essentially normal for every $p>d$.

Proof. Let $N_{1}, \ldots, N_{n}$ denote the $H_{d}^{2}$ submodules generated by $F_{1}, \ldots, F_{n}$ respectively, and let $V_{1}, \ldots, V_{n}$ denote the $\mathbb{C}^{r}$ subspaces spanned by $X_{1}, \ldots, X_{n}$ respectively. Let $M$ denote the $r H_{d}^{2}$ submodule generated by the set (5.1), and let $M_{1}, \ldots, M_{n}$ denote the $r H_{d}^{2}$ submodules

$$
M_{i}=N_{i} \otimes V_{i}, \quad 1 \leq i \leq n .
$$

Then Theorem 5.4 implies that each of the submodules $N_{1}, \ldots, N_{n}$ is p-essentially normal for every $p>d$, and Proposition 4.12 implies that the family $\left\{N_{1}, \ldots, N_{n}\right\}$ is perpendicular. Note that $M=\overline{M_{1}+\ldots+M_{n}}$. Hence by Theorem [5.2, $M$ is also $p$-essentially normal for every $p>d$.

The next theorem is Arveson's main result from Arv05. Shalit also gave a proof of this result in Sha11] using his results on stable division. The methods introduced here provide a new and simple proof. Recall that a monomial of $r H_{d}^{2}$ is an element in $r \mathbb{C}[z]$ of the form $z^{\alpha} \otimes \xi$ for some $\alpha$ in $\mathbb{N}_{0}^{d}$ and $\xi$ in $\mathbb{C}^{r}$.

Theorem 5.6 (Arveson). Every submodule of $r H_{d}^{2}$ generated by monomials is $p$ essentially normal for every $p>d$.

Proof. Let $N$ be a submodule of $r H_{d}^{2}$ generated by monomials, say $z^{\alpha_{1}} \otimes \xi_{1}, \ldots, z^{\alpha_{n}}$ $\otimes \xi_{n}$ in $r \mathbb{C}[z]$ for $\alpha_{1}, \ldots, \alpha_{n}$ in $\mathbb{N}_{0}^{d}$. Let $N_{1}, \ldots, N_{n}$ denote the $r H_{d}^{2}$ submodules generated by $z^{\alpha_{1}}, \ldots, z^{\alpha_{n}}$ respectively, and let $V_{1}, \ldots, V_{n}$ denote the one-dimensional subspaces of $\mathbb{C}^{r}$ spanned by $\xi_{1}, \ldots, \xi_{n}$ respectively. Then Theorem 5.3 implies that the submodules $N_{1}, \ldots, N_{n}$ are $p$-essentially normal for every $p>d$, and Proposition 4.13 implies that the family $\left\{N_{1}, \ldots, N_{n}\right\}$ is perpendicular. Note that $N=\overline{N_{1}+\ldots+N_{n}}$. Hence by Theorem 5.2, $N$ is also $p$-essentially normal for every $p>d$.

Recall Shalit's result from [Sha11] that a submodule generated by polynomials in two variables has the stable division property. Shalit used this result to prove the next theorem that these submodules are essentially normal. However, starting from Proposition 3.10, we can also view Shalit's proof of the stable division property for 
these submodules as a method for establishing essential decomposability. In this case, the methods introduced here provide a new proof.

Theorem 5.7 (Shalit). Let $F$ be a set of homogeneous polynomials. Suppose that there is a subset $Z$ of $\left\{z_{1}, \ldots, z_{d}\right\}$, of size at most 2 , such that $F \subseteq \mathbb{C}[Z]$. Then the $H_{d}^{2}$ submodule generated by $F$ is $p$-essentially normal for every $p>d$.

Proof. Let $N$ denote the $H_{d}^{2}$ submodule generated by $F$, and let $\left\{p_{1}, \ldots, p_{n}\right\}$ be a Groebner basis consisting of homogeneous polynomials that generates $N$. Let $N_{1}, \ldots, N_{n}$ denote the $H_{d}^{2}$ submodules generated by $p_{1}, \ldots, p_{n}$ respectively. Then by Theorem 5.3. $N_{1}, \ldots, N_{n}$ are $p$-essentially normal for every $p>d$. Note that the polynomials $p_{1}, \ldots, p_{n}$ belong to $\mathbb{C}[Z]$. Hence by Proposition $3.10, N=N_{1}+\ldots+$ $N_{n}$, and $N$ is $p$-essentially decomposable for every $p>d$. It follows from Theorem 3.3 that $M$ is $p$-essentially normal for every $p>d$.

The next result is new. It implies the essential normality of a large new class of submodules of $r H_{d}^{2}$.

Theorem 5.8. Let $F_{1}, \ldots, F_{n}$ be sets of homogeneous polynomials that each generate p-essentially normal submodules of $H_{d}^{2}$ for $p>d$. Suppose that there are disjoint subsets $Z_{1}, \ldots, Z_{n}$ of $\left\{z_{1}, \ldots, z_{d}\right\}$ such that

$$
F_{i} \subseteq \mathbb{C}\left[Z_{i}\right], \quad 1 \leq i \leq n .
$$

Let $X_{1}, \ldots, X_{n}$ be arbitrary sets of vectors in $\mathbb{C}^{r}$. Then the $r H_{d}^{2}$ submodule generated by the set of vector-valued polynomials

$$
\left\{p \otimes \xi \mid p \in F_{i}, \quad \xi \in X_{i}, 1 \leq i \leq n\right\}
$$

is p-essentially normal.

Proof. Let $N_{1}, \ldots, N_{n}$ denote the $H_{d}^{2}$ submodules generated by $F_{1}, \ldots, F_{n}$ respectively, and let $V_{1}, \ldots, V_{n}$ denote the $\mathbb{C}^{r}$ subspaces spanned by $X_{1}, \ldots, X_{n}$ respectively. Let $M$ denote the $r H_{d}^{2}$ submodule generated by the set (5.2), and let $M_{1}, \ldots, M_{n}$ denote the $r H_{d}^{2}$ submodules

$$
M_{i}=N_{i} \otimes V_{i}, \quad 1 \leq i \leq n .
$$

The submodules $N_{1}, \ldots, N_{n}$ are $p$-essentially normal by assumption, and Proposition 4.14 implies that the family $\left\{N_{1}, \ldots, N_{n}\right\}$ is perpendicular. Note that $M=$ $\overline{M_{1}+\ldots+M_{n}}$. Hence by Theorem 5.2, $M$ is also $p$-essentially normal.

Replacing the use of Proposition 4.14 in the proof of Theorem 5.8 with Proposition 4.17 and Proposition 4.18 respectively, we immediately obtain the following strengthened results.

Theorem 5.9. Let $F_{1}, \ldots, F_{n}$ be sets of homogeneous polynomials that each generate $p$-essentially normal submodules of $H_{d}^{2}$ for $p>d$. Suppose that the sets

$$
\left\{\partial^{\alpha}(p)|| \alpha \mid=\operatorname{deg}(p)-1, \alpha \in \mathbb{N}_{0}^{d}, p \in F_{i}\right\}, \quad 1 \leq i \leq n,
$$

are mutually orthogonal. Let $X_{1}, \ldots, X_{n}$ be arbitrary sets of vectors in $\mathbb{C}^{r}$. Then the $\mathrm{rH}_{d}^{2}$ submodule generated by the set of vector-valued polynomials

$$
\left\{p \otimes \xi \mid p \in F_{i}, \xi \in X_{i}, 1 \leq i \leq n\right\}
$$

is p-essentially normal. 
Theorem 5.10. Let $F_{1}, \ldots, F_{n}$ be sets of homogeneous polynomials that each generate $p$-essentially normal submodules of $H_{d}^{2}$ for $p>d$. Suppose that the sets

$$
\left\{(\nabla p)(\lambda) \mid \lambda \in \mathbb{C}^{d}, p \in F_{i}\right\}, \quad 1 \leq i \leq n,
$$

are mutually orthogonal. Let $X_{1}, \ldots, X_{n}$ be arbitrary sets of vectors in $\mathbb{C}^{r}$. Then the $r H_{d}^{2}$ submodule generated by the set of vector-valued polynomials

$$
\left\{p \otimes \xi \mid p \in F_{i}, \xi \in X_{i}, 1 \leq i \leq n\right\}
$$

is p-essentially normal.

The next theorem follows from a combination of the results above.

Theorem 5.11. Let $F_{1}, \ldots, F_{n}$ be sets of homogeneous polynomials in $\mathbb{C}[z]$. Suppose that there are disjoint subsets $Z_{1}, \ldots, Z_{n}$ of $\left\{z_{1}, \ldots, z_{d}\right\}$, each of size at most 2 , such that

$$
F_{i} \subseteq \mathbb{C}\left[Z_{i}\right], \quad 1 \leq i \leq n .
$$

Let $X_{1}, \ldots, X_{n}$ be arbitrary sets of vectors in $\mathbb{C}^{r}$. Then the $H_{d}^{2} \otimes \mathbb{C}^{r}$ submodule generated by the set of vector-valued polynomials.

$$
\left\{p \otimes \xi \mid p \in F_{i}, \xi \in X_{i}, 1 \leq i \leq n\right\}
$$

is $p$-essentially normal for every $p>d$.

Proof. This follows immediately from Theorem 5.7 and Theorem 5.8 .

Example 5.12. For every even $d \geq 1$ and $n \geq 1$, let $N$ denote the $H_{d}^{2}$ submodule generated by the set of polynomials $p_{1}, \ldots, p_{d / 2}$, where

$$
\begin{aligned}
p_{1}\left(z_{1}, \ldots, z_{d}\right) & =z_{1}^{n}+z_{1}^{n-1} z_{2}+\ldots+z_{1} z_{2}^{n-1}+z_{2}^{n} \\
& \vdots \\
p_{d / 2}\left(z_{1}, \ldots, z_{d}\right) & =z_{d-1}^{n}+z_{d-1}^{n-1} z_{d}+\ldots+z_{d-1} z_{d}^{n-1}+z_{d}^{n}
\end{aligned}
$$

Then Theorem 5.11 implies that $N$ is $p$-essentially normal for every $p>d$.

\section{ACKNOWLEDGEMENTS}

The author is grateful to Ken Davidson and Orr Shalit for many helpful comments and suggestions. The author would also like to thank the anonymous referee for suggestions which greatly improved the exposition.

\section{REFERENCES}

[Arv98] William Arveson, Subalgebras of $C^{*}$-algebras. III. Multivariable operator theory, Acta Math. 181 (1998), no. 2, 159-228, DOI 10.1007/BF02392585. MR 1668582 (2000e:47013)

[Arv00] William Arveson, The curvature invariant of a Hilbert module over $\mathbf{C}\left[z_{1}, \cdots, z_{d}\right]$, J. Reine Angew. Math. 522 (2000), 173-236, DOI 10.1515/crll.2000.037. MR.1758582 (2003a:47013)

[Arv02] William Arveson, The Dirac operator of a commuting d-tuple, J. Funct. Anal. 189 (2002), no. 1, 53-79, DOI 10.1006/jfan.2001.3827. MR.1887629 (2003a:47014)

[Arv05] William B. Arveson, p-summable commutators in dimension d, J. Operator Theory 54 (2005), no. 1, 101-117. MR2168861 (2007b:47012)

[Arv07] William Arveson, Quotients of standard Hilbert modules, Trans. Amer. Math. Soc. 359 (2007), no. 12, 6027-6055 (electronic), DOI 10.1090/S0002-9947-07-04209-2. MR2336315 (2009i:46101) 
[BGM10] C. Badea, S. Grivaux, and V. Müller, The rate of convergence in the method of alternating projections, Algebra i Analiz 23 (2011), no. 3, 1-30, DOI 10.1090/S1061-0022-201201202-1; English transl., St. Petersburg Math. J. 23 (2012), no. 3, 413-434. MR2896163 (2012j:46032)

[Car06] Enrico Carlini, Reducing the number of variables of a polynomial, Algebraic geometry and geometric modeling, Math. Vis., Springer, Berlin, 2006, pp. 237-247, DOI 10.1007/978-3-540-33275-6_15. MR2279854 (2007i:12010)

[CLS92] David Cox, John Little, and Donal O'Shea, Ideals, varieties, and algorithms, Undergraduate Texts in Mathematics, Springer-Verlag, New York, 1992. An introduction to computational algebraic geometry and commutative algebra. MR1189133 (93j:13031)

[DRS11] Kenneth R. Davidson, Christopher Ramsey, and Orr Moshe Shalit, The isomorphism problem for some universal operator algebras, Adv. Math. 228 (2011), no. 1, 167-218, DOI 10.1016/j.aim.2011.05.015. MR2822231(2012j:46083)

[DRS12] K. Davidson, C. Ramsey, O. Shalit, Operator algebras for analytic varieties, Preprint (2012), arXiv:1201.4072.

[Dou06] Ronald G. Douglas, Essentially reductive Hilbert modules, J. Operator Theory 55 (2006), no. 1, 117-133. MR2212024 (2007h:47014)

[Esc11] Jörg Eschmeier, Essential normality of homogeneous submodules, Integral Equations Operator Theory 69 (2011), no. 2, 171-182, DOI 10.1007/s00020-010-1809-4. MR2765583 (2012c:47019)

[Fri37] Kurt Friedrichs, On certain inequalities and characteristic value problems for analytic functions and for functions of two variables, Trans. Amer. Math. Soc. 41 (1937), no. 3, 321-364, DOI 10.2307/1989786. MR 1501907

[FW71] P. A. Fillmore and J. P. Williams, On operator ranges, Advances in Math. 7 (1971), 254-281. MR0293441 (45 \#2518)

[GW08] Kunyu Guo and Kai Wang, Essentially normal Hilbert modules and K-homology, Math. Ann. 340 (2008), no. 4, 907-934, DOI 10.1007/s00208-007-0175-2. MR.2372744 (2009c:47006)

[Rez93] Bruce Reznick, An inequality for products of polynomials, Proc. Amer. Math. Soc. 117 (1993), no. 4, 1063-1073, DOI 10.2307/2159535. MR.1119265 (93e:11058)

[Sha11] Orr Moshe Shalit, Stable polynomial division and essential normality of graded Hilbert modules, J. Lond. Math. Soc. (2) 83 (2011), no. 2, 273-289, DOI 10.1112/jlms/jdq054. MR2776637 (2012e:46120)

School of Mathematics and Statistics, Carleton University, 1125 Colonel By Drive, Ottawa, Ontario, Canada K1S 5B6

E-mail address: mkennedy@math.carleton.ca 